\title{
Lingual lobe of right lung- a rare variation
}

\author{
Mohanty S.R ${ }^{1}$, Yadav $\mathrm{B}^{2}$. \\ Kalinga Institute of Medical Sciences, Bhubaneswar. Smruti Rekha Mohanty, MS \\ Assistant Professor ,Department of Anatomy, Kalinga Institute of Medical Sciences, \\ KIIT University ,Patia, Bhubaneswar-751024.Dist-Khurda ,State-Odisha INDIA
}

\begin{abstract}
Knowledge of variations of lobar pattern of lung is important for identification of bronchopulmonary segments. It is of great clinical significance for surgeons and radiologists. Normally the right lung has two fissures, one oblique and one horizontal and three lobes, upper, middle and lower lobes. Many authors have described the lobar variations on imaging techniques but a few studies have reported in gross anatomy. The present case highlights an abnormal lingual lobe of the right lung which was detected incidentally during the undergraduate dissection. Also it highlights the abnormal pattern of oblique and horizontal fissures.
\end{abstract}

Keywords: right lung, horizontal fissure, oblique fissure, lingual lobe, variation

\section{Introduction}

Incomplete fissures of lung are a common observations, but are often misinterpreted on radiographs and computed scans. Lobar anatomy and broncho-pulmonary segments can be appreciated better with knowledge of variations. Classically the right lung is divided into three lobes namely upper, middle and lower by two fissures, an oblique and a horizontal. The oblique fissure runs downwards, thereby meeting the inferior border of the lung at a distance of approximately $7.5 \mathrm{~cm}$ behind anterior end (standring). The horizontal fissure, passes from the anterior margin into the oblique fissure to separate a wedge shaped middle lobe from the upper lobe. It passes at the level of midaxillary line to the anterior border of the lung at the level of sternal end of $4^{\text {th }}$ costal cartilage.

The present case describes a S-shaped oblique fissure and incomplete curved horizontal fissure with upward convexity and a pyriform shaped lingual lobe in between the two fissures.

\section{Case Report}

During routine dissection classes of thoracic region for the undergraduate students, an unusual lobar variation of right lung was observed. The oblique fissure of right lung cut the posterior border of lung, $6 \mathrm{cms}$ from the apex of the lung. Another curved fissure commences at a distance of $16.5 \mathrm{cms}$ from the posterior border. The curved fissure divided the oblique fissure into a cranial part and a caudal part. The curvature of the oblique fissure was convexo-concave. The oblique fissure could be traced upto the lower end of hilum. The curved fissure was well defined in its proximal part but at a distance of $3.5 \mathrm{cms}$ from the anterior border of right lung it could not be delineated. A lingual lobe was observed between these two fissures. The maximum height of the lingual lobe was $10.5 \mathrm{cms}$ and maximum width was $6.5 \mathrm{cms}$. The distance between the lower end of oblique fissure and the anterior border of right lung was $5 \mathrm{cms}$. The distance between the anterior end of curved fissure and the inferior border was $4 \mathrm{cms}$.

\section{Discussion}

The right lung is divided into superior, middle and inferior lobes by two fissures, an oblique and a horizontal (1). The oblique fissure separates the inferior lobe from middle and superior lobes and corresponds closely to the left oblique fissure although it is less vertical, crossing the inferior border of the lung about $7.5 \mathrm{cms}$ behind its anterior end. On the posterior border it is level with the spine of 4th thoracic vertebra or slightly lower. It descends across the 5th intercostal space and follows the 6th rib to the 6th costochondral junction(2).

The short horizontal fissure separate the superior and middle lobes.It passes from the oblique fissure, near the mid axillary line, horizontally forwards to the anterior border of the lung, level with the sternal end of the 4th costal cartilage, then passes backwards to the hilum on the mediastinal surface. The small middle lobe is thus cuneiform and includes some of the costal surface, the lower part of the anterior border and the anterior part of the base of the lung (3).

During foetal life as the lung develops, the spaces of fissures that separate individual bronchopulmonary buds or segments become obliterated except along the oblique and horizontal fissures (4). Incomplete fissures could be result of abnormal obliterations of these fissures, partially or completely.

Knowledge of variations of incomplete fissures and lobes of lung is important for preoperative planning strategy for pulmonary lobectomy and segmental resection of lung.(5). Incomplete fissure is also a 
cause for post operative air leakage (6). Incomplete fissures are also responsible for altering the spread of any lung disease(7).

Anatomical knowledge of fissures of lung helps in differential diagnosis of chest diseases in case of extension of fluid into an incomplete major fissure or spread of diseases through different pathways (8). Incomplete fissures as recorded in the present case are often mistaken for the appearance of pleural effusion. (9). The incomplete horizontal fissure in the present case also described an abnormal upward convex course and along with sinuous course of lower part of oblique fissure, also there is variation in the middle lobe with tongue like projection. The incompleteness, thick sections and orientation of fissures may obscure them during radiographic procedure and increase clinicians dilemma (10).

It is proven fact that lymphatics of the lung drain centripetally from the pleura towards hilum (11). In the present case, an abnormal course of horizontal fissure and lower part of oblique fissure would mean an anomalous or altered course of visceral pleura, thereby changing the course and arrangement of lymphatic drainage. An incomplete horizontal fissure as observed in the present case could be mistaken for a scar or a pleural line made visible by pneumothorax (12).

\section{Conclusion}

Considering the clinical importance of such variations, we as an anatomist opine that prior anatomical knowledge for the presence of variations in fissures, lobes and broncho-pulmonary segments may be essential for cardiothoracic surgeons and radiologists.

\section{References}

[1]. Standring S. Gray’s Anatomy. 40 Edition. Churchill Livingstone, 2008. pp-993

[2]. Walker WS \& Craig SR. A proposed anatomical classification of the pulmonary fissures. J R Coll Surg Edinburgh 1997; 42(4):2334.

[3]. Godwin JD \& Tarver RD. Accessory Fissures of the Lung. AJR 1985; 144(1):39-47.

[4]. Modgil V, Das S, Suri R K. Anomalous lobar pattern of right lung- A case report. Int J Morphol 2006; 24(1):5-6.

[5]. Meenakshi S, Manjunath K, Balasubramanyam V. Morphological Variations of the Lung Fissures and lobes. The Indian J of Chest Diseases \& Allied Sciences 2004; 46(3): 179-82.

[6]. Das S, Latiff AA, Othman FB et al. Topographical anatomy of anomalous oblique fissure and lingual of the lung. Braz J Morph Sci 2007; 24(3):155-56.

[7]. Rosse C, Gaddum-Rosse P. Hollinshed's Textbook of Anatomy. Philadelphia: Lipincott-Raven; 1997: 441-61.

[8]. Dandy WE. Incomplete pulmonary interlobar fissure sign. Radiology 1978; 1289(1):21-5.

[9]. [9].

[10]. Ellis H. Clinical Anatomy. A revision and applied anatomy for clinical students. 1 edh ed. USA: Blackwell Publishing, 2002.

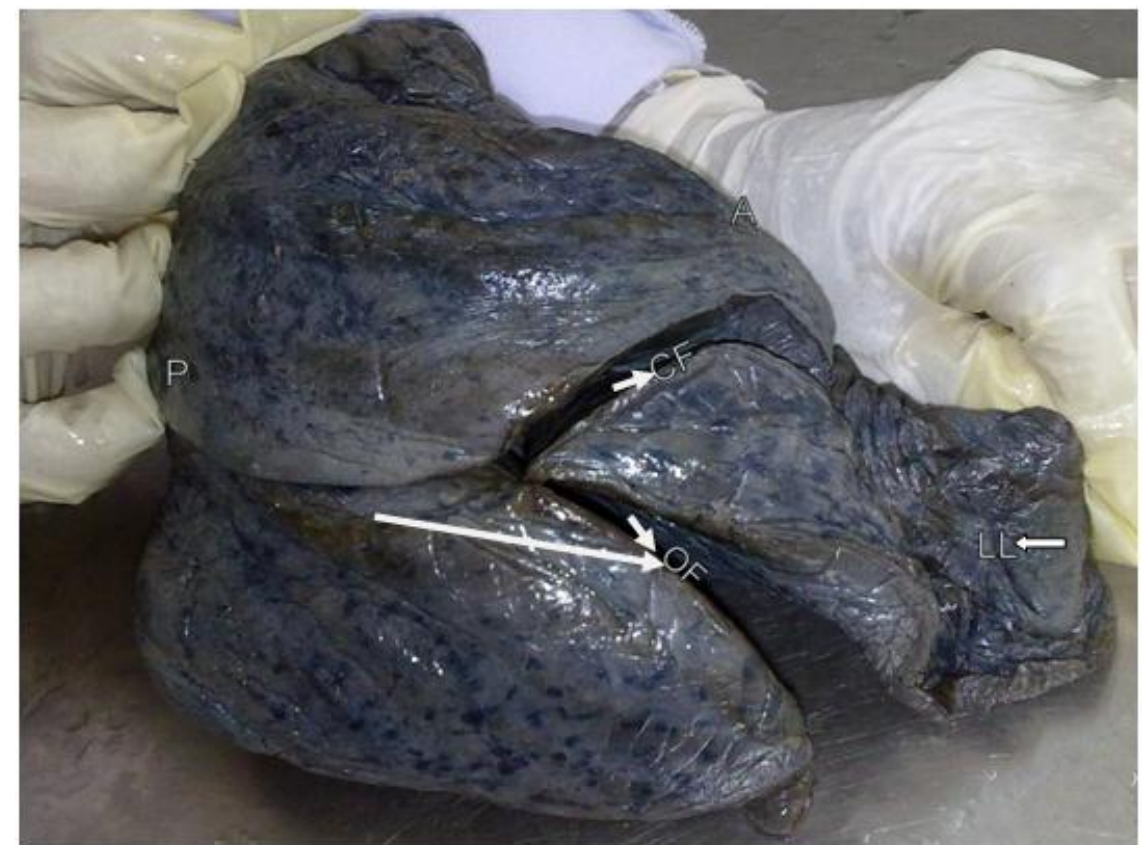

Figure1: Right lung showing the lingual lobe and incomplete curved horizontal fissure.(OF-Oblique fissure,CFCurved fissure,A-Anterior border,P-Posterior border,LL-Lingual lobe) 


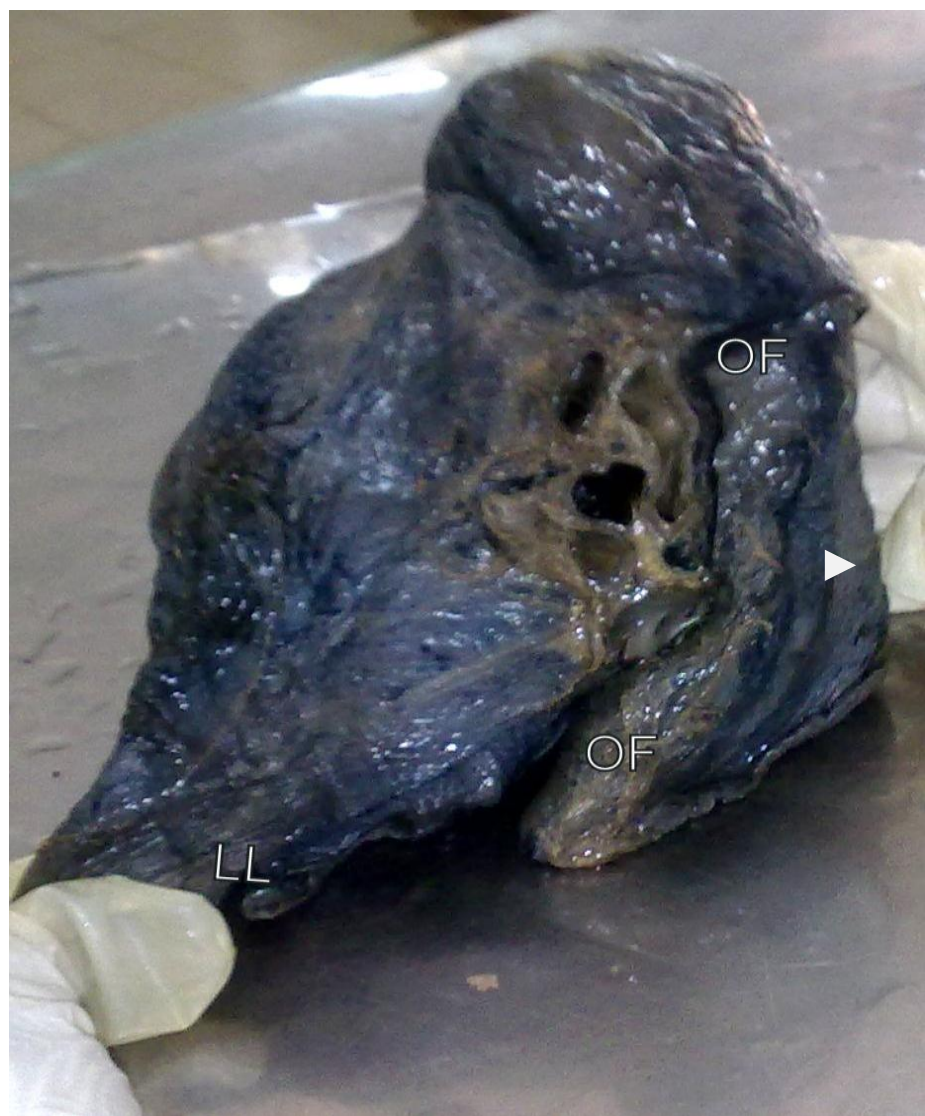

Figure2: Medial surface of Right lung showing Lingual lobe.(OF-Oblique fissure,LL-Lingual lobe) 